\title{
Postoperative Aortic Neck Dilation: Myth or Fact?
}

\author{
A.S. Ribner, MD ${ }^{1}$ A.K. Tassiopoulos, MD, FACS ${ }^{1}$ \\ ${ }^{1}$ Division of Vascular Surgery, Stony Brook University Hospital, Stony \\ Brook, New York \\ Address for correspondence A.K. Tassiopoulos, MD, FACS, Division of \\ Vascular Surgery, Stony Brook University Hospital, Stony Brook, NY \\ Int J Angiol 2018;27:110-113. \\ 11794 (e-mail: Apostolos.Tassiopoulos@stonybrookmedicine.edu).
}

\author{
Abstract \\ Keywords \\ - endovascular repair \\ - abdominal aortic \\ aneurysm \\ - aneurysm \\ - aortic valve disease \\ - endograft placement \\ - repair \\ - artery
}

The abdominal aorta is the most common site of an aortic aneurysm. The visceral and most proximal infrarenal segment (aneurysm neck) are usually spared and considered more resistant to aneurysmal degeneration. However, if an abdominal aortic aneurysm (AAA) is left untreated, the natural history of the aortic neck is progressive dilatation and shortening. This may have significant implications for patients undergoing endovascular repair of AAAs (EVAR) as endograft stability and integrity of the repair are dependent on an intact proximal seal zone. Compromised seal zones, caused by progressive diameter enlargement and foreshortening of the aortic neck, may lead to distal endograft migration, type la endoleak, aortic sac repressurization, and, ultimately, aortic rupture.
The abdominal aorta is the most common site of an aortic aneurysm. The visceral and most proximal infrarenal segment (aneurysm neck) are usually spared and considered more resistant to aneurysmal degeneration. However, if an abdominal aortic aneurysm (AAA) is left untreated, the natural history of the aortic neck is progressive dilatation and shortening. ${ }^{1}$ This may have significant implications for patients undergoing endovascular repair of AAAs (EVAR) as endograft stability and integrity of the repair are dependent on an intact proximal seal zone. Compromised seal zones, caused by progressive diameter enlargement and foreshortening of the aortic neck, may lead to distal endograft migration, type Ia endoleak, aortic sac repressurization, and, ultimately, aortic rupture.

\section{Is There Evidence of Progressive Neck Dilatation?}

Postoperative structural changes at the proximal neck of AAAs have been noted for years and their occurrence is unsurprising, given the already compromised integrity of the aneurysmal vessel wall. Illig et al reported significant dilatation of the AAA neck in about one-third of patients undergoing open surgical repair (OSR), ${ }^{2}$ and Falkensammer et al calculated the annual rate of dilatation to be $0.16 \mathrm{~mm}$ in the infrarenal segment and $0.18 \mathrm{~mm}$ in the suprarenal segment following OSR. ${ }^{3}$ This supports the theory of progressive structural deterioration but is of limited clinical importance for patients who have undergone OSR. On the other hand, progressive aortic neck diameter enlargement is more concerning in patients undergoing EVAR with self-expanding endografts, which represent the vast majority of commercially approved devices. The generally accepted belief is that proximal neck dilatation leading to type la endoleaks is due to outward radial forces exerted by stent grafts on the aortic wall and, as long-term follow-up data of patients after EVAR accumulates, cases of aortic neck dilatation (AND) beyond the nominal proximal diameter of the endograft are identified (-Fig. 1). Setting a threshold of $2 \mathrm{~mm}$ to define AND, Oberhuber et al reported an increase in diameter after both EVAR and OSR both at the infrarenal level (22.3 and 19.6\%, respectively; $p=0.87$ ), and at the suprarenal level (20.4 and $30.4 \%$, respectively; $p=0.26$ ) with the former occurring more frequently after OSR and the latter after EVAR. ${ }^{4}$ Interestingly, there were no significant differences between the dilatation rates at both levels when comparing EVAR with OSR. This raises a question regarding how much additional stress the endograft outward radial force poses to the aneurysm neck. The authors suggested that neck dilation is published online

May 23, 2018
Copyright $\odot 2018$ by Thieme Medical Publishers, Inc., 333 Seventh Avenue, New York, NY 10001, USA. Tel: +1(212) 584-4662.
DOI https://doi.org/ 10.1055/s-0038-1649516. ISSN 1061-1711. 

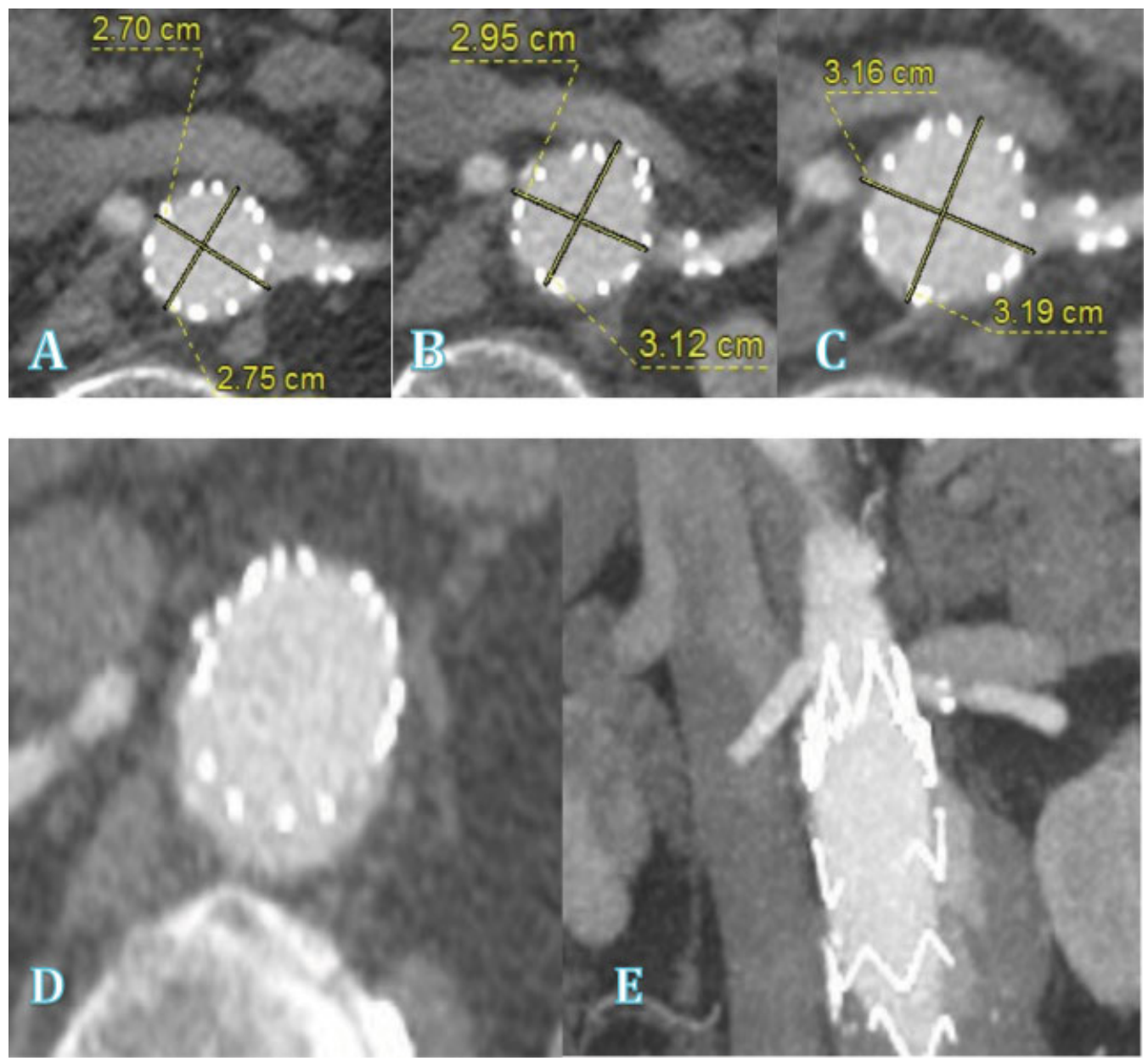

Fig. 1 Progressive dilatation of the aortic neck at the level of the left (lowest) renal artery captured in follow-up computed tomography (CT) angiograms obtained at 2 months (A), 13 months (B), and 27 months (C) from the time of endograft implantation, with development of type la endoleak (D) and distal endograft migration (E).

likely "multifactorial" and an intrinsic element of the progression of aortic disease, rather than due to any one discrete process. Several other reports also suggest the process to be much more complex than pure mechanical outward force. In fact, Georgakarakos et al notes that endovascular treatment of AAAs should not be considered a single step intervention based on preoperative anatomical features alone, but rather that the aorta continues to remodel around the endograft for years after implantation. ${ }^{5}$ This concept is essential for the modern vascular surgeon to consider when planning the initial AAA intervention and any subsequent adjunctive procedures to enhance longevity of the repair.

When considering loss of seal at the proximal neck, two major factors come into play: neck dilatation and endograft distal migration. These may occur independently or, indeed, be caused by one another. There is no doubt that endografts without active fixation may migrate distally even in the absence of significant neck dilatation. More commonly, however, a gradual outpouching of the aortic wall causes loss of endograft apposition and distal displacement resulting in pressure transmission onto the adjacent unlined wall and, ultimately, further dilatation. It had previously been observed $^{6,7}$ and then proven via computational stimulation by Georgakarakos et al that the factors most responsible for augmentation of displacement forces are the inlet (neck) diameter and the inlet-to-outlet ratio, with greater neck angulations also appearing to influence maintenance of an intact proximal seal zone. In fact, almost all AAAs repaired with endografts undergo some degree of proximal neck deterioration, clinically significant or not, with the one exception of extremely favorable anatomies (small diameter necks and little to no angulation). These are becoming increasingly uncommon in current clinical practice, as EVARs outside the manufacturer's instructions for use (IFU) now represent a significant percentage of all endovascular AAA repairs. Several reviews support the observations by Georgakarakos et al and show a significantly higher rate of proximal seal zone complications and type la endoleaks in patients with "hostile" neck anatomy when compared to those with "favorable" anatomy. ${ }^{8}$ Hostile anatomic characteristics include short aortic neck length $(<15 \mathrm{~mm})$, large $(>29 \mathrm{~mm}$ ) preoperative aortic neck diameter, high suprarenal and/or infrarenal neck angulation, reverse taper or conical neck configuration, and larger amount of neck thrombus and calcium. Using a 3-mm threshold, we recently reported that post-EVAR dilatation of the aortic neck $5 \mathrm{~mm}$ 
distal to the lowest renal artery in patients with hostile neck anatomy was seen in $12.5 \%$ of all patients 1 month postEVAR, and in $8.1 \%$ of patients between 1 month and 1 year following endograft implantation. ${ }^{9}$ Independent risk factors predicting AND in our study included: larger preoperative aortic diameter at the level of the lowest renal artery, use of endografts with suprarenal stents, and higher degree of endograft oversizing. Kret et al also reported that endograft oversizing was the most significant factor linked to AND. ${ }^{10}$ Aggressive oversizing ( $>25 \%$ ) is frequently chosen to compensate for adverse neck characteristics such as short or conical necks. Multiple device-specific studies documented this result as well and noted that the maximum diameter reached by most dilated necks was the same diameter as that of the implanted device. ${ }^{11,12}$ The hypothesis of these studies is that the wall merely dilates to accommodate the oversized graft and then stops once it is fully incorporated. Although this observation may seem to support the notion that outward radial forces play the greatest role in proximal neck dilation, another possible explanation is that of suboptimal apposition of the aggressively oversized endograft to the aortic wall and more extensive endograft pleating that may create an environment of local microleaks. These, in turn, lead to increased pressure on the aortic wall at the proximal seal zone that may be equally, if not more, important in the progressive enlargement of the proximal neck diameter. Tsilimparis et al, in their investigation of the effects of the Zenith endograft on neck dilation, observed continuous progression of neck diameter growth at the 30-day, 2-year, and 5-year mark, with the greatest rates of growth occurring in the immediate postperiod and, to a lesser extent, at 3 years. $^{13}$ The early steep growth curve was attributed to the initial accommodation of the aortic wall to the newly implanted graft and all subsequent change to the ongoing degeneration of the diseased aortic wall. This would explain why and how proximal neck dilation occurs both in endovascular and after open repair, albeit at different rates. ${ }^{14}$

\section{Can Aortic Neck Dilatation Be Prevented?}

Most reports on post-EVAR AND have studied proximal neck diameter changes after EVAR with self-expanding stents (SES), but one notable study looked at AND incidence after repair using balloon expandable stent (BES) grafts. ${ }^{15}$ The authors looked at AND following implantation of a homemade aortouniiliac stent graft consisting of a polytetrafluoroethylene (PTFE) graft affixed to a proximal Palmaz stent. This graft was chosen for implantation in patients who were excluded from commercially available devices due to unfavorable neck anatomy (acute neck angulation $>60^{\circ}$ or short necks $<15 \mathrm{~mm}$ ). There were no incidences of AND or endograft migration reported. The authors suggested two possible explanations: First, the lack of stent incorporation into the aortic wall with $\mathrm{SES}^{16}$ as opposed to the Palmaz stent which becomes a part of the aortic wall with greater success. Second, the fact that SES are subject to repetitive motion within the aorta leading to material "fatigue." This ultimately results in loss of tensile strength and resultant outpouching causing dilation of the neck wall. Another potential consideration is the accuracy of endograft deployment in the proximal neck. Zarins et al has suggested that the further below the renal arteries a stent graft is deployed, the more likely the adjacent wall is to undergo subsequent dilation. Hence, with precise deployment of BES, the graft may be deployed much closer to the renal arteries without fear of obstruction. ${ }^{17}$ Similar results were observed with the VIDatascope graft in a small series of patients with difficult neck anatomy at an average follow-up period of 11 months. ${ }^{18}$ There are, however, two notable factors that may confound these conclusions. Generally, BES are only oversized by $5 \%$, whereas SES are oversized by 10 to $20 \%$. As explained previously, this would greatly affect the rate of AND. It is also conceivable that proximal seal may be better with BES. In addition, all EVARs with BES had an aortouniiliac configuration. Prior studies have shown that downward force at the bifurcation plays a role in distal migration, but this factor is limited with uniiliac devices and so it is difficult to reasonably compare the two types of grafts.

In 2013, TriVascular released its Ovation stent graft, which contained a unique feature specifically designed to enhance the proximal seal zone. The "O rings," are a set of two hollow rings within the graft main body at the proximal end that are filled with polymer after deployment to expand to the exact size and shape of the aortic lumen and create a tight seal; they can even establish seal with adjacent thrombus or calcification and in challenging cases of reversed taper anatomy. Most importantly, once the rings have been deployed during the initial repair, they do not expand further, thereby preventing the progressive outward radial force on the wall of the aortic neck observed with other stent grafts. The theoretical benefit of such a design was supported in a study by de Donato et al which reported freedom from type Ia endoleak at 3 years to be $98 \%{ }^{19}$ However, the length of follow-up in that study was not long enough to capture the progressive changes in aortic neck diameter that occur as a result of disease progression alone, particularly when one considers the presence of the two long suprarenal bare metal stents that are designed to provide main body fixation. The structure of this endograft is such that even a small increase in aortic neck diameter at the site of the $O$ rings would result in loss of seal and a type Ia endoleak. Therefore, long-term follow-up is essential to determine the rate of AND and proximal seal zone compromise with this endograft.

Considering the incidence and clinical significance of AND and endograft migration, the use of prophylactic proximal fixation devices during the initial repair should be strongly considered-particularly in patients with hostile neck anatomies. Grisafi et al in 2011 first published results for use of multiple proximal fixation adjuncts (angioplasty, extension cuffs, and uncovered stenting) for assisted primary patency. ${ }^{20}$ In the mid-2000s, the HeliFx Endoanchor fixation device was released in the market as a novel tool that improves endograft fixation and seal at the proximal neck. The driving force behind its development was the need for a device that could be deployed endovascularly with the precision and strength of an open surgical suture. ${ }^{21}$ It has 
been suggested that when Endoanchors are appropriately deployed, they improve endograft fixation and apposition to the aortic wall, thus improving proximal seal after EVAR. Muhs et al compared two well-matched EVAR patient cohorts (with and without use of Endoanchors) and reported significantly higher rates of aortic sac regression in patients who underwent EVAR with Endoanchors, but no significant differences in the rate of type Ia endoleaks. ${ }^{22}$ This supports the notion that Endoanchor use improves proximal endograft seal. ANCHOR is a prospective, non-randomized, dualarm, multicenter, postmarket registry of the real-world use of the Heli-FX system with Endoanchors. The registry has over 800 EVAR patients enrolled worldwide without any reports of endograft migration, indicating the efficacy of the device in enhancing endograft fixation. We recently found Endoanchors to be an independent predictor of prophylaxis against AND; our hypothesis is that once the aortic neck diameter reaches the nominal endograft diameter, the Endoanchors keep the aortic wall attached to the endograft, acting as a stabilizing structure and preventing further dilatation of the neck. It is conceivable that decreasing endograft oversizing with concomitant prophylactic use of Endoanchors could be an effective strategy for preventing AND in both short and long term and should be considered in EVAR patients with longer life expectancy.

\section{Conclusions}

Aortic neck dilatation with most commercially available selfexpanding endografts is well-documented, multifactorial, and an important parameter when planning endovascular AAA repair, particularly in patients with "hostile" neck anatomy. Endograft selection, degree of oversizing, use of adjuncts that enhance fixation and sealing, and the option of OSR should be strongly considered especially in patients with longer life expectancy.

\section{References}

1 Propper BW, Rasmussen TE, Jones WT, Gifford SM, Burkhardt GE, Clouse WD. Temporal changes of aortic neck morphology in abdominal aortic aneurysms. J Vasc Surg 2010;51(05):1111-1115

2 Illig KA, Green RM, Ouriel K, Riggs P, Bartos S, DeWeese JA. Fate of the proximal aortic cuff: implications for endovascular aneurysm repair. J Vasc Surg 1997;26(03):492-499, discussion 499-501

3 Falkensammer J, Oldenburg WA, Biebl M, et al. Abdominal aortic aneurysm neck remodeling after open aneurysm repair. J Vasc Surg 2007;45(05):900-905

4 Oberhuber A, Schwarz A, Hoffmann MH, Klass O, Orend K-H, Mühling B. Influence of different self-expanding stent-graft types on remodeling of the aortic neck after endovascular aneurysm repair. J Endovasc Ther 2010;17(06):677-684

5 Georgakarakos E, Argyriou C, Schoretsanitis N, et al. Geometrical factors influencing the hemodynamic behavior of the AAA stent grafts: essentials for the clinician. Cardiovasc Intervent Radiol 2014;37(06):1420-1429

6 Jim J, Rubin BG, Geraghty PJ, Criado FJ, Fajardo A, Sanchez LAA. A 5-year comparison of EVAR for large and small aortic necks. J Endovasc Ther 2010;17(05):575-584

7 Stanley BM, Semmens JB, Mai Q et al. Evaluation of patient selection guidelines for endoluminal AAA repair with the Zenith Stent-Graft: the Australasian experience. J Endovasc Ther 2001;8 (05):457-464

8 Antoniou GA, Georgiadis GS, Antoniou SA, Kuhan G, Murray D. A meta-analysis of outcomes of endovascular abdominal aortic aneurysm repair in patients with hostile and friendly neck anatomy. J Vasc Surg 2013;57(02):527-538

9 Tassiopoulos AK, Monastiriotis S, Jordan WD, Muhs BE, Ouriel K, De Vries JP. Predictors of early aortic neck dilatation after endovascular aneurysm repair with EndoAnchors. J Vasc Surg 2017;66 (01):45-52

10 Kret MR, Tran K, Lee JT. Change in aortic neck diameter after endovascular aortic aneurysm repair. Ann Vasc Surg 2017;43: $115-120$

11 Monahan TS, Chuter TA, Reilly LM, Rapp JH, Hiramoto JS. Longterm follow-up of neck expansion after endovascular aortic aneurysm repair. J Vasc Surg 2010;52(02):303-307

12 Soberón AB, de Garcia MM, Möll GG, Vigil BR, Krauel MA, AlvarezSala Walter R. Follow-up of aneurysm neck diameter after endovascular repair of abdominal aortic aneurysms. Ann Vasc Surg 2008;22(04):559-563

13 Tsilimparis N, Dayama A, Ricotta JJ II. Remodeling of aortic aneurysm and aortic neck on follow-up after endovascular repair with suprarenal fixation. J Vasc Surg 2015;61(01):28-34

14 Oberhuber A, Buecken M, Hoffmann M, Orend K-H, Mühling BM. Comparison of aortic neck dilatation after open and endovascular repair of abdominal aortic aneurysm. J Vasc Surg 2012;55(04): 929-934

15 Malas MB, Ohki T, Veith FJ, et al. Absence of proximal neck dilatation and graft migration after endovascular aneurysm repair with balloon-expandable stent-based endografts. J Vasc Surg 2005;42(04):639-644

16 Guidoin R, Marois Y, Douville Y, et al. First-generation aortic endografts: analysis of explanted Stentor devices from the EUROSTAR Registry. J Endovasc Ther 2000;7(02):105-122

17 Zarins CK, Bloch DA, Crabtree T, Matsumoto AH, White RA, Fogarty TJ. Stent graft migration after endovascular aneurysm repair: importance of proximal fixation. J Vasc Surg 2003;38(06): 1264-1272, discussion 1272

18 Kolvenbach R, Pinter L, Cagiannos C, Veith FJ. Remodeling of the aortic neck with a balloon-expandable stent graft in patients with complicated neck morphology. Vascular 2008;16(04):183-188

19 de Donato G, Setacci F, Bresadola L, et al; TriVascular Ovation Italian Study. Aortic neck evolution after endovascular repair with TriVascular Ovation stent graft. J Vasc Surg 2016;63(01):8-15

20 Grisafi JL, Rahbar R, Nelms J, et al. Challenging neck anatomy is associated with need for intraoperative endovascular adjuncts during endovascular aortic aneurysm repair (EVAR). Ann Vasc Surg 2011;25(06):729-734

21 Deaton DH. Improving proximal fixation and seal with the HeliFx Aortic EndoAnchor. Semin Vasc Surg 2012;25(04):187-192

22 Muhs BE, Jordan W, Ouriel K, Rajaee S, de Vries JP. Matched cohort comparison of endovascular abdominal aortic aneurysm repair with and without EndoAnchors. J Vasc Surg 2017; S0741-5214(17) 32502-8. Doi: 10.1016/j.jvs.2017.10.059 